Bernhardt, Anna; Fiedler, Patrique; Fonseca, Carlos; Gonçalves, Ricardo;

Haueisen, Jens:

Novel dry electrode EEG headbands for home use: comparing performance and comfort

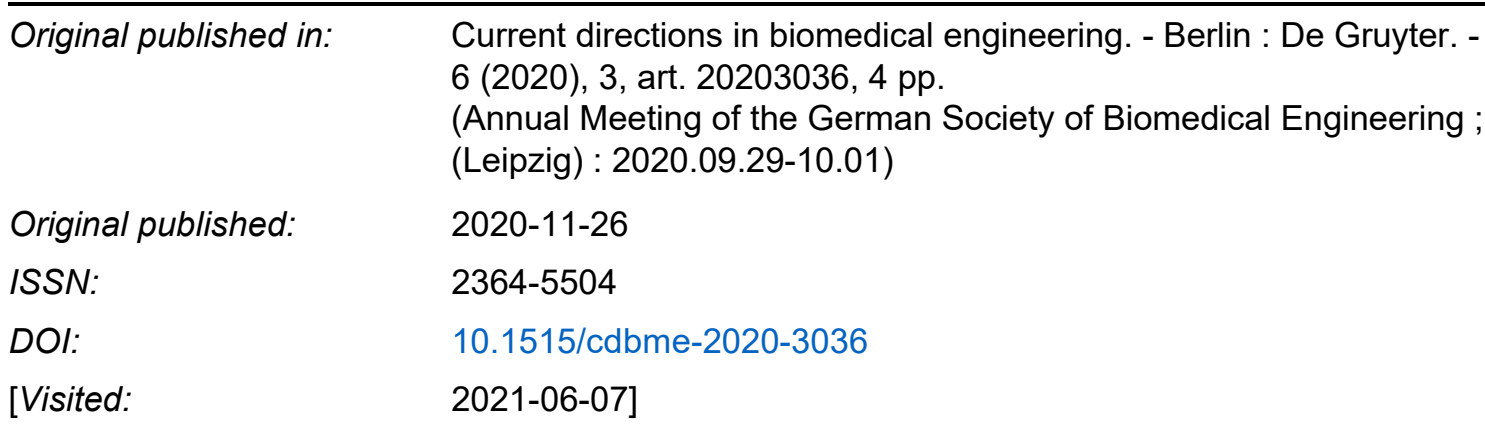




\section{Novel dry electrode EEG headbands for home use: Comparing performance and comfort}

\begin{abstract}
Monitoring brain activity at home using electroencephalography (EEG) is an increasing trend for both medical and non-medical applications. Gel-based electrodes are not suitable due to the gel application requiring extensive preparation and cleaning support for the patient or user. Dry electrodes can be applied without prior preparation by the patient or user.

We investigate and compare two dry electrode headbands for EEG acquisition: a novel hybrid dual-textile headband comprising multipin and multiwave electrodes and a neoprene-based headband comprising hydrogel and spidershaped electrodes. We compare the headbands and electrodes in terms of electrode-skin impedance, comfort, electrode offset potential and EEG signal quality.

We did not observe considerable differences in the power spectral density of EEG recordings. However, the hydrogel electrodes showed considerably increased impedances and offset potentials, limiting their compatibility with many EEG amplifiers. The hydrogel and spider-shaped electrodes required increased adduction, resulting in a lower wearing comfort throughout the application time compared to the novel headband comprising multipin and multiwave electrodes.
\end{abstract}

Keywords: biopotential electrode, bioelectric signal, electroencephalography, EEG, neurofeedback, brain computer interfaces, BCI

https://doi.org/10.1515/cdbme-2020-3036

\section{INTRODUCTION}

Monitoring and modulation of brain activity in mobile and home-use environments are becoming increasingly important for neurofeedback, brain-computer interfaces (BCI), at-home diagnostics and therapy for patients with neurological pathologies [1]. Electroencephalography (EEG) is the most commonly used modality for mobile brain monitoring due to state-of-the-art compact, lightweight electronics for amplifiers [1]. Conventional gel-based electrodes are not suitable for outof-the-lab environments. Dry electrodes reduce the preparation time not requiring the application of conductive electrolyte gel, making EEG acquisition cleaner, simpler and more cost-effective. Dry electrodes can be applied by the patient or user without the need for assistance.

Diagnostic and research applications often require highdensity electrode montages to provide sufficient spatial resolution for e.g., full-head activity monitoring, source localization and/or connectivity analysis [2]. In contrast, research in BCI and neurofeedback aim to determine an optimal and low number of electrode positions [3], reducing preparation time and product costs.

We investigate two concepts for dry electrode EEG headbands comprising four types of commercially available dry electrode technologies. We compare the headbands and electrodes in terms of electrode-skin impedance, EEG signal quality, application time and wearing comfort.

\section{MATERIALS AND METHODS}

\footnotetext{
${ }^{*}$ Corresponding author: Jens Haueisen: Institute of Biomedical Engineering and Informatics Technische Universität IImenau, Gustav-Kirchhoff-Strasse 2, IImenau, Germany Patrique Fiedler: eemagine Medical Imaging Solutions $\mathrm{GmbH}$, Berlin, Germany, and Institute of Biomedical Engineering and Informatics, Technische Universität IImenau, Germany

Anna Bernhardt, Ricardo Gonçalves: Institute of Biomedical Engineering and Informatics, Technische Universität IImenau, Gustav-Kirchhoff-Str. 2, IImenau, Germany

Carlos Fonseca: Faculdade de Engenharia da Universidade do Porto, Porto, Portugal
}

\subsection{Electrode types}

Design requirements for electrode shapes and flexibility vary depending on the head position the electrodes are intended to be applied to. The shape and material of electrodes applied to hairy head regions must allow easy application through the hair to establish a direct, stable contact with the scalp. The most commonly proposed shapes include multiple fingers or 
pins which individually pass through the hair, and different shapes, diameters and orientation [4]. While adaptivity of the electrode materials and thus geometry is of paramount importance for electrode-skin contact stability and comfort, electrode pins at hairy head regions need to be stiff enough to go through the hair, but not to bend or slide on top of it. Anterior and mastoid head positions are free of extensive, dense hair and therefore require only a wave-like shape to accommodate few, individual strands of hair. Due to the increased pressure sensitivity of frontal head regions, a soft electrode material is important for comfort at frontal electrode positions [5].

We compared an overall number of four electrode types: dry multipin and multiwave electrodes (ANT neuro bv, Hengelo, Netherlands), and dry spider-shaped and hydrogel electrodes (Cognionics Inc., San Diego, USA). An overview of the principal design parameters of all compared electrodes is provided in Table 1 .

The multiwave and the hydrogel electrodes are intended for frontal head positions and have an overall diameter of $15.5 \mathrm{~mm}$ (multiwave, ANT neuro bv) and $17 \mathrm{~mm}$ (hydrogel, Cognionics Inc.), respectively. Both electrodes exhibit a wavelike shape of the contact area.

Multipin and spider-shaped electrodes are intended for hairy central, temporal, parietal and occipital positions. The multipin electrode (ANT neuro bv) investigated in this study comprises an overall diameter of $25.5 \mathrm{~mm}$ and 79 pins. The spider-like electrode (Cognionics Inc.) has a diameter of $25 \mathrm{~mm}$ with 8 pins. The multipin and multiwave electrodes are based on a polyurethane substrate of shore hardness A80, subsequently coated with silver/silver-chloride $(\mathrm{Ag} / \mathrm{AgCl})$ using a multi-phase electroless plating procedure that enables more than 250 applications. The spider electrodes comprise a conductive carbon-doped polymer and an additional silver (Ag) paint at the contact points. The hydrogel electrodes (Cognionics Inc.) comprise a top layer of a hydrogel on a rigid electrode and polymer holder.

\subsection{Headbands}

Two headbands were compared: a novel, highly flexible headband combining neoprene and sports textile, and a neoprene-only headband. While the novel hybrid headband contained multipin and multiwave electrodes, the neopreneonly headband was equipped with spider-shaped and hydrogel electrodes. Both headbands were equipped with electrodes at positions AF3, AF4, PO3 and PO4 as well as a GND electrode at position Fpz. An overview of the principal designs of both compared headbands is shown in Figure 1.

The novel hybrid headband is based on a double layer design made of stretchable sports textiles. The inner layer provides structural stability but remains stretchable, and is based on neoprene textile. The outer layer is made of highly flexible, rapid-drying fabric, which is also used in commercial EEG caps (waveguard, ANT neuro). All electrodes are fixated using specifically designed mechanical fixation clips. Electrical connection is established by soldering. Shielded / coaxial cables are implemented from the connector to the electrodes. The aforementioned textile layers fully cover the mechanical fixation clips and the coaxial cables. A clip mechanism above the left ear enables adjustment of the headband length according to the individual's head circumference. A flat-snap cable is integrated behind the right ear for the reference electrode.

Table 1: Key parameters and characteristics of the compared electrode types

\begin{tabular}{|c|c|c|c|c|}
\hline \multirow{2}{*}{$\begin{array}{l}\text { Intended electrode } \\
\text { position and name }\end{array}$} & \multicolumn{2}{|c|}{ anterior, frontal } & \multicolumn{2}{|c|}{ central, temporal, parietal, and occipital } \\
\hline & Hydrogel & Multiwave & Spider-shape & Multipin \\
\hline $\begin{array}{l}\text { Manufacturer } \\
\text { Picture }\end{array}$ & Cognionics Inc. & ANT neuro bv & Cognionics Inc. & ANT neuro bv \\
\hline Substrate material & Rigid polymer & Flexible polyurethane & $\begin{array}{l}\text { Flexible, carbon-doped } \\
\text { polymer }\end{array}$ & Flexible polyurethane \\
\hline Contact material & Hydrogel & $\mathrm{Ag} / \mathrm{AgCl}$ coating & Ag paint & $\mathrm{Ag} / \mathrm{AgCl}$ coating \\
\hline Overall diameter & $17 \mathrm{~mm}$ & $15.5 \mathrm{~mm}$ & $25 \mathrm{~mm}$ (fully spread) & $25.5 \mathrm{~mm}$ \\
\hline Pin number & Flat, wavy surface & 30 & 8 & 79 \\
\hline Pin top diameter & n.a. & $1.5 \mathrm{~mm}$ & $1.5 \mathrm{~mm}$ & $1 \mathrm{~mm}$ \\
\hline $\begin{array}{c}\text { Overall contact } \\
\text { surface }\end{array}$ & ca. $201 \mathrm{~mm}^{2}$ & ca. $90 \mathrm{~mm}^{2}$ & ca. $28 \mathrm{~mm}^{2}$ & ca. $123 \mathrm{~mm}^{2}$ \\
\hline
\end{tabular}


The neoprene-only headband is based on two identical layers of neoprene textile with integrated female snap fasteners for both mechanical and electrical electrode integration. Unshielded cables are used within the headband, running between both textile layers. An adapter cable using shielded coaxial cable was manufactured for connecting the headband to the amplifier. The adapter cable integrates a flatsnap cable placed behind the right ear for connecting the reference electrode. A Velcro mechanism is integrated in the back of the head to allow adjustment of the headband length. a)

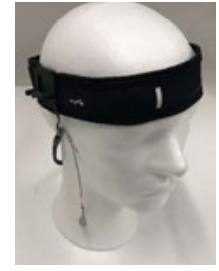

b)

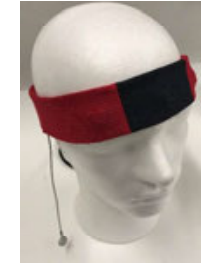

evaluated immediately after application of the headband as well as $10 \mathrm{~min}, 30 \mathrm{~min}$ and $60 \mathrm{~min}$ later.

Multiple EEG sequences were recorded, including 2 minutes of resting state EEG (eyes open), and 2 minutes with eyes closed. EEG signals were postprocessed by application of a $36 \mathrm{~dB}$ Butterworth bandpass filter with cutoff frequencies at 1 and $40 \mathrm{~Hz}$. Analysis sequences were selected by using one window of 30 seconds length per recording sequence, skipping the first 10 seconds of the recording in order to minimize the eventual influence of filter artefacts. Power spectral density (PSD) was calculated using the Welch estimation method.

\section{RESULTS}

\subsection{Application time and comfort}

Figure 1: Photos of the two compared headbands: a) the neoprene-only headband comprising hydrogel and spider-shaped electrodes, b) the hybrid headband comprising multiwave and multipin electrodes.

\subsection{Test setup and paradigm}

Both headbands were applied for sequential measurements in a randomized sequence on overall 10 volunteers with an average age of $25.4 \pm 2.6$ years. All volunteers were males with a head circumference of $58.7 \pm 1.5 \mathrm{~cm}$ and hair length of $5 \pm 1.9 \mathrm{~cm}$. All volunteers reported healthy psychological, neurological and dermatological condition as well as no known record of drug abuse.

The volunteers were instructed to clean their hair on the morning before the EEG recordings. The reference electrode was placed at the right mastoid position using a self-adhesive electrode (Kendall ECG electrodes H124SG, Covidien LLC, Mansfield, USA). The skin at the reference position was cleaned with alcohol prior to electrode application. No further skin preparation was performed. The headbands were tightened as much as required to achieve impedances below $1 \mathrm{MOhm}$ at all electrode positions.

For EEG and impedance measurements, a referential mobile amplifier (eego sports, ANT Neuro bv) was used. The amplifier provided active shielding for each channel. EEG data was recorded at 1024 samples/sec.

The volunteers were asked for comfort evaluation on a scale ranging from 1 to 10 , wherein 1 corresponds to maximum comfort, while 10 corresponds to pain requiring taking off the headband immediately. The comfort was
No considerable differences in terms of application time were observed between the two headbands. Both headbands were applied to the volunteers within less than $3 \mathrm{~min}$.

The comfort rating of the volunteers varied at different application times. The respective mean comfort ratings at 0 , 10,30 and 60 minutes after application were $2.6 \pm 1.1,2.8 \pm$ $1,5 \pm 2$ and $5.7 \pm 2.2$ for the hybrid headband. The corresponding comfort ratings of the neoprene-only headband were $4.3 \pm 1.9,4.4 \pm 2,7.3 \pm 1.7$, and $8.9 \pm 1.2$. Throughout the application time, the comfort rating of the novel hybrid headband comprising multipin and multiwave electrodes was superior in comparison to the neoprene-only headband comprising hydrogel and spider-like electrodes.

\subsection{Electrode-skin impedance}

Figure 2 shows the topographic distribution of the electrodeskin impedances, measured immediately before the EEG recordings.

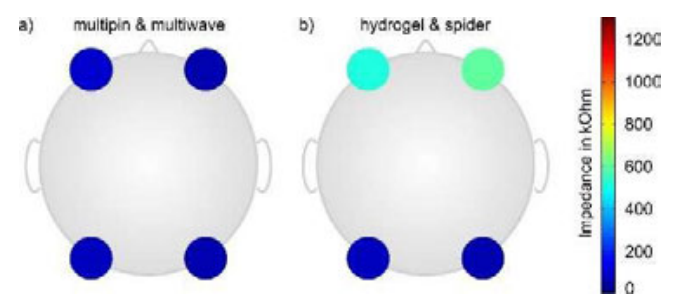

Figure 2: Topographic distribution of the electrode-skin impedance before the EEG recordings, calculated over all 10 subjects: a) the hybrid headband equipped with multiwave and multipin electrodes b) the neoprene-only headband equipped with hydrogel and spidershape electrodes. 
The mean impedance over all volunteers and electrodes for the hybrid headband was $80 \mathrm{kOhm} \pm 22 \mathrm{kOhm}$, in comparison to $316 \mathrm{kOhm} \pm 293 \mathrm{kOhm}$ for the neoprene-only headband. The topographic distribution shows that the electrodes in the neoprene-only headband exhibited higher electrode-skin impedances than the ones in the hybrid headband. In particular, the impedances at the frontal electrode positions were considerably increased for the hydrogel electrodes. Furthermore, the variability of the impedances for the neoprene-only headband was increased.

\subsection{Electrode offset potential}

For the neoprene-only headband a strong separation between the frontal (hydrogel) electrodes and the occipital (spider-shaped) electrodes was observed. The offset potentials at the frontal electrodes varied around $300 \mathrm{mV}$, which is the boundary of the dynamic range of many state-of-the-art EEG amplifiers. For the occipital electrodes, the potential was closely distributed within the range of -50 to $50 \mathrm{mV}$. In contrast, for the hybrid headband, all electrodes showed a distribution within the -50 to $50 \mathrm{mV}$ interval.

\subsection{EEG power spectral density}

The average PSD calculated over all electrodes and volunteers for $30 \mathrm{sec}$ of resting state EEG with eyes open and $30 \mathrm{sec}$ of with closed eyes is shown in Figure 3. For the recordings of both EEG headbands and all types of electrodes, no considerable difference was visible. Alpha activity was clearly pronounced around $10 \mathrm{~Hz}$ for the eyes closed recordings.

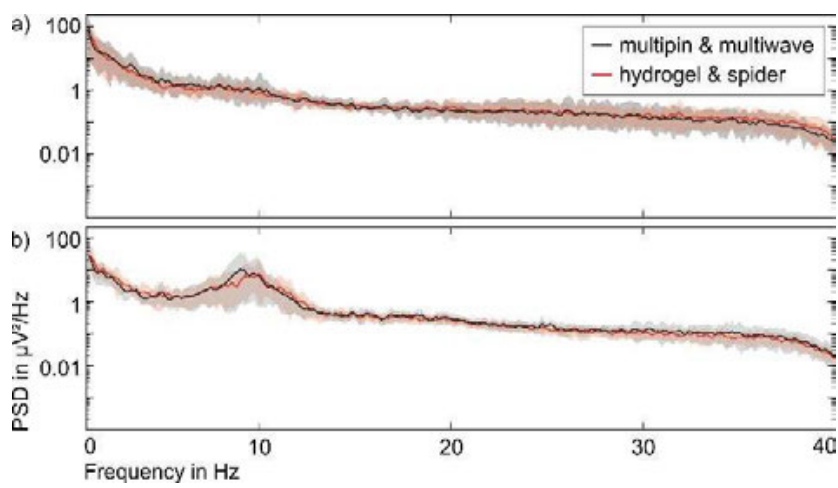

Figure 3: Power spectral density of EEG recordings of $30 \mathrm{sec}$ length with a) eyes open and b) eyes closed, averaged over all 10 subjects. Solid lines indicate mean, transparent areas indicate standard deviation.

\section{DISCUSSION}

We compared two headbands comprising commercially available dry electrodes for rapid at-home EEG. The headbands provided equivalent signal quality when used with a commercially available referential amplifier. Prior investigations indicated that the impedance of dry electrodes decreases with longer time of application to the skin [6]. The headbands might require less adduction for the EEG recordings than indicated by the impedance measurement right after application, consequently improving comfort.

The high offset potentials observed for the hydrogel electrodes require a large dynamic range and will compromise their compatibility with many amplifiers. Previous investigations also showed high levels of offset potentials to correlate with increased electrode drift [4], further limiting the applicability of hydrogel electrodes. The compatibility of the hydrogel electrodes will be further limited by the high electrode-skin impedance level. The very high input impedance ( $>1 \mathrm{GOhm}$ ) and active shielding technology of the amplifier used in this study contributed to the high signal quality. An increased influence of artifacts should to be expected when using unshielded cables.

In conclusion, the results of this study demonstrate the applicability of the multipin and multiwave electrode technologies for EEG acquisition. The performance and comfort of the novel hybrid headband and its multipin and multiwave electrodes may contribute to an increased applicability for repeated and longterm at-home applications for e.g., neurofeedback and BCI.

\section{References}

[1] Debener S, Minow F, Emkes R, Gandras K, de Vos M. How about taking a low-cost, small, wireless EEG for a walk? Psychophys 2012;49(11):1617-1621.

[2] Puce A, Hämäläinen M. A Review of Issues Related to Data Acquisition and Analysis in EEG/MEG Studies. Brain Sci 2017;7(6):58.

[3] Mihajlovic V, Garcia-Molina G, Peuscher J. Dry and WaterBased EEG Electrodes in SSVEP-Based BCI Applications. Proceedings of the BIOSTEC 2012 2013;357:23-40.

[4] Fiedler P, Haueisen J, Jannek D, Griebel S, Zentner L, Vaz $\mathrm{F}$, et al. Comparison of three types of dry electrodes for electroencephalography. Acta IMEKO 2014;3(3):33-37.

[5] Fiedler P, Mühle R, Griebel S, Pedrosa P, Fonseca C, Vaz F, et al. Contact Pressure and Flexibility of Multipin Dry EEG Electrodes. IEEE Trans. Neural. Syst. Rehabil. Eng 2018;26(4):750-757.

[6] Fiedler P, Pedrosa P, Griebel S, Fonseca C, Vaz F, Supriyanto E, et al. Novel Multipin Electrode Cap System for Dry Electroencephalography. Brain Topo 2015;28:647-656. 